\title{
Ramsey interference in a multilevel quantum system
}

\author{
J. P. Lee, ${ }^{1,2}$ A. J. Bennett, ${ }^{1, *}$ J. Skiba-Szymanska, ${ }^{1}$ D. J. P. Ellis, ${ }^{1}$ I. Farrer,${ }^{3}$ D. A. Ritchie, ${ }^{4}$ and A. J. Shields ${ }^{1}$ \\ ${ }^{1}$ Toshiba Research Europe Limited, Cambridge Research Laboratory, 208 Science Park, Milton Road, Cambridge, CB4 OGZ, United Kingdom \\ ${ }^{2}$ Engineering Department, University of Cambridge, 9 J. J. Thomson Avenue, Cambridge, CB3 OFA, United Kingdom \\ ${ }^{3}$ Department of Electronic \& Electrical Engineering, University of Sheffield, Mappin Street, Sheffield, S1 3JD, United Kingdom \\ ${ }^{4}$ Cavendish Laboratory, Cambridge University, J. J. Thomson Avenue, Cambridge, CB3 OHE, United Kingdom \\ (Received 22 July 2015; revised manuscript received 19 January 2016; published 4 February 2016)
}

\begin{abstract}
We report Ramsey interference in the excitonic population of a negatively charged quantum dot measured in resonant fluorescence. Our experiments show that the decay time of the Ramsey interference is limited by the spectral width of the transition. Applying a vertical magnetic field induces Zeeman split transitions that can be addressed by changing the laser detuning to reveal two-, three-, and four-level system behavior. We show that under finite field the phase-sensitive control of two optical pulses from a single laser can be used to prepare both population and spin states simultaneously. We also demonstrate the coherent optical manipulation of a trapped spin in a quantum dot in a Faraday geometry magnetic field.
\end{abstract}

DOI: 10.1103/PhysRevB.93.085407

\section{INTRODUCTION}

Ramsey interferometry has found use in cesium atomic clocks and in investigations of the quantum nature of the electromagnetic field [1,2]. Applying this technique to solid state systems promises new applications and insights [3-5] including accurate coherence time measurements of solid state quantum systems [6], the implementation of simple quantum algorithms [7], and magnetometers with nanoscale spatial resolution [8].

Prior measurements of Ramsey interference (RI) between exciton population levels in quantum dots (QDs) have used one of two techniques. In some works $[3,9,10]$ the laser is resonant with a transition and measurement of the population is made in photocurrent, which necessitates the destruction of the exciton, shortening its lifetime. Alternatively, optical readout of the population has been made when excitation occurs via a phonon-assisted transition [9] or a two-photon transition [4] and thus the laser can be removed by spectral filtering.

Here we demonstrate a direct measurement of RI in the resonant fluorescence (RF) of a QD. The measurements are performed on the negative trion transition and dependent on the external magnetic field and laser energy, we observe two-, three-, and four-level system behavior. This leads to a rich variety of interferograms with exponential decays, beats, and oscillations which reveal information about the coherence of the exciton and the trapped charge. Our results show how controlling the time delay between subsequent pulses from a single laser allows us to prepare a population superposition simultaneously with a spin state superposition. We believe that these results will open new possibilities to control and measure the dynamics of spin qubits in mesoscopic semiconductor systems. As a metrology technique RI gives unparalleled precision in measurement of the emitter's local environment $[8,11]$.

Ramsey interference (RI) fringes are the result of the oscillation in the population of a state as a function of the time delay between two $\frac{\pi}{2}$ pulses. The first pulse creates a superposition

*anthony.bennett@crl.toshiba.co.uk between the ground and excited state-interpreted as a $\frac{\pi}{2}$ rotation of the Bloch vector about the $x$ axis of the Bloch sphere [Fig. 1(a)]. The system then undergoes free evolution-the Bloch vector precesses about the equator of the Bloch sphere [Fig. 1(b)] at a frequency determined by the energy difference between the ground and excited states. The second $\frac{\pi}{2}$ pulse

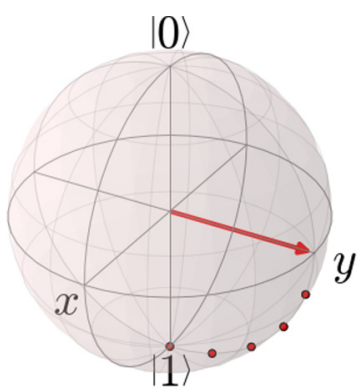

(a)

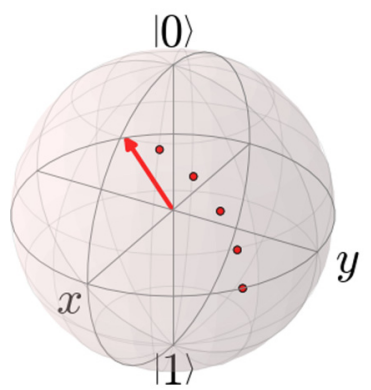

(c)

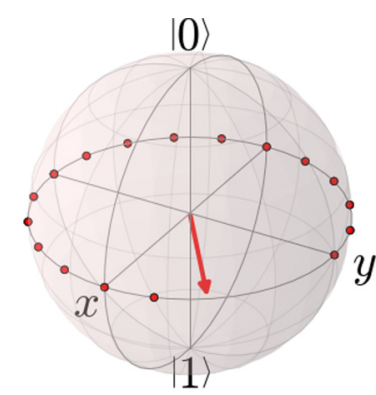

(b)

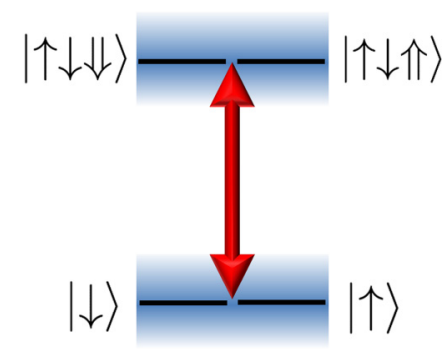

(d)
FIG. 1. (a) A $\frac{\pi}{2}$ pulse creates a coherent superposition of the excited and ground states; the Bloch vector is rotated by $\frac{\pi}{2}$ about the $x$ axis. (b) The system undergoes free evolution; the Bloch vector precesses about the $z$ axis. (c) A second $\frac{\pi}{2}$ pulse maps the system into the excited or ground state dependent on the time delay between the pulses; the Bloch vector is rotated by $\frac{\pi}{2}$ about the $x$ axis. (d) An illustration of the energy levels of the negatively charged QD at zero magnetic field. (Bloch spheres were produced with [12].) 


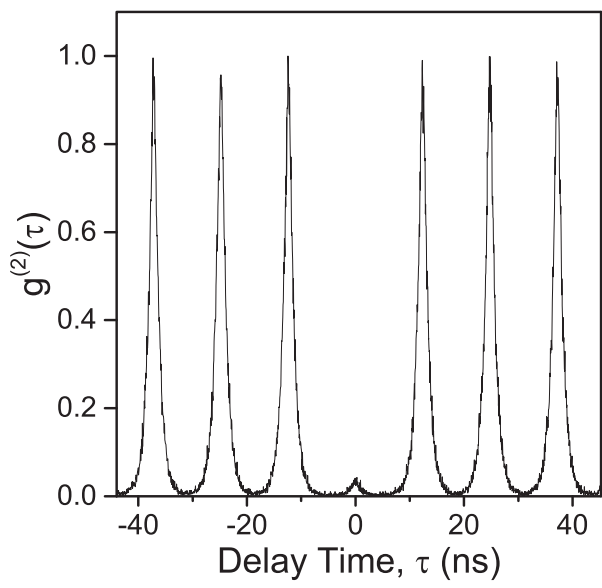

(a)

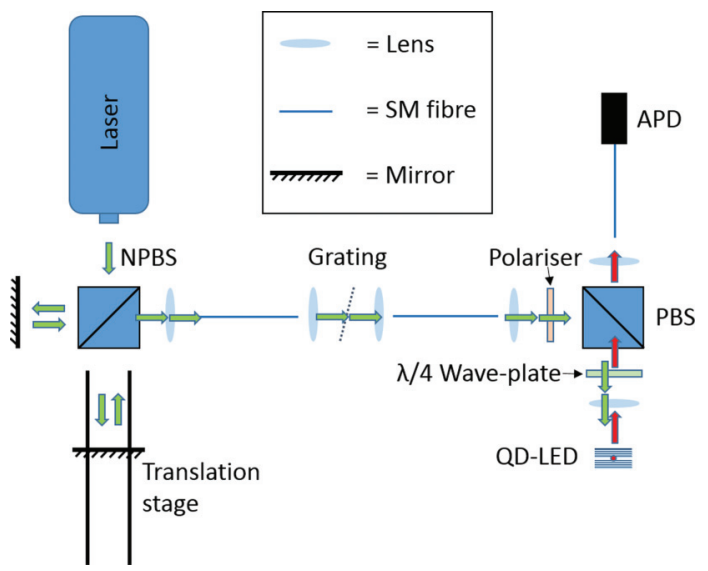

(d)

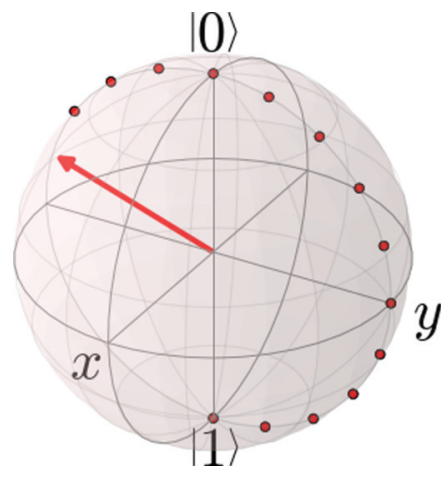

(b)

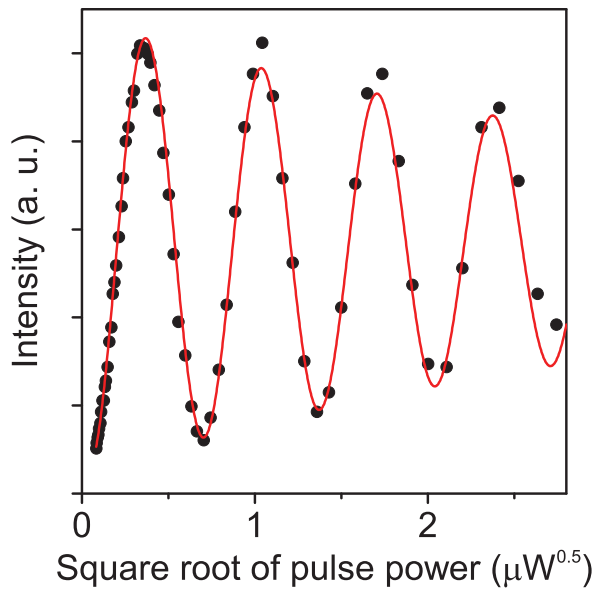

(c)

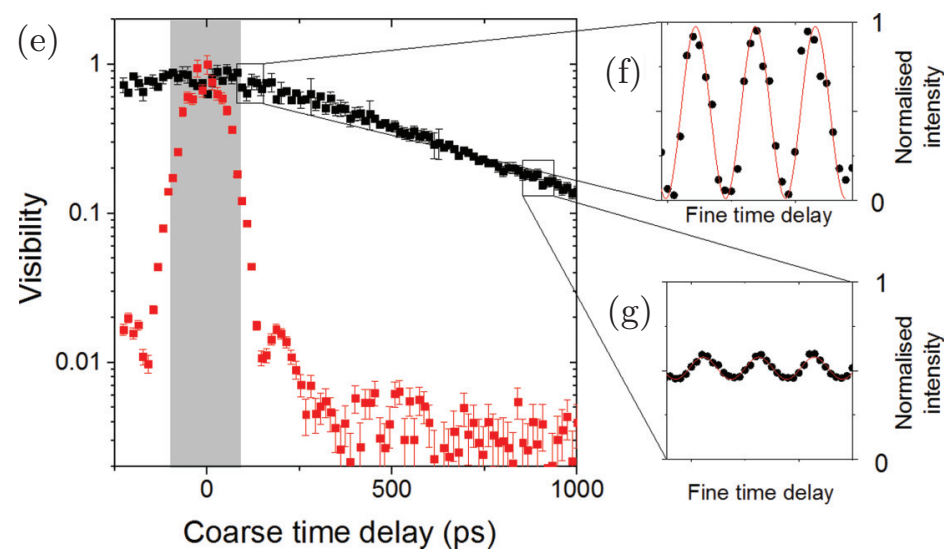

FIG. 2. (a) A pulsed Hanbury-Brown and Twiss $g^{(2)}(\tau)$ measurement. (b) The rotation of the Bloch vector about the $x$ axis. (c) Rabi oscillations. (d) An illustration of our experimental setup for performing RI. (e) (Red) Interference visibility of input laser pulses. (Black) The visibility of the Ramsey fringes as a function of pulse separation. (f) Emission intensity as a function of fine time delay at a short pulse separation (50 ps). (g) Emission intensity as a function of fine time delay at a longer pulse separation (1000 ps).

then maps the system to the ground or excited state dependent on the time delay of the two pulses. Again, this is interpreted as a rotation of the Bloch vector about the $x$ axis [Fig. 1(c)]. At zero magnetic field the energy levels of a negatively charged QD are spin independent, so the dot behaves as a two-level system [Fig. 1(d)].

\section{EXPERIMENT}

The InGaAs dot used was embedded in the undoped region of an LED planar microcavity cooled to $5 \mathrm{~K}$. The device was held below the threshold voltage so no electroluminescence was seen, but the dot was loaded with an electron. Excitation was performed using $57 \mathrm{ps}$ resonant laser pulses (generated by filtering 3 ps pulses from a mode locked Ti:sapphire laser with a grating) with an $80 \mathrm{MHz}$ repetition rate. The pulses are short relative to the exciton lifetime of $959 \pm 2$ ps. Cross-polarization filtering (with an extinction ratio of $10^{7}$ ) was used to separate the linearly polarized excitation light from the circularly polarized emitted light. The emitted light was not spectrally or temporally filtered ensuring that the spectral density function of the emitted photons remained intact. This prevents an artificial increase in the decay time of the Ramsey fringe visibility.

\section{RESULTS}

To determine that we are exciting a single quantum emitter, we perform a Hanbury-Brown and Twiss measurement and record $g^{(2)}(0)=0.06$ [Fig. 2(a)]. The measurement is performed using $0.7 \pi$ pulses. The peak at $\tau=0$ is less than 0.5 , which indicates that the detected light is primarily composed of single photons.

Next, we demonstrate that we can coherently manipulate the excitonic population, which we interpret as rotating the Bloch vector about the $x$ axis [Fig. 2(b)]. We do this by recording the emitted light intensity as a function of the square root of pulse power and observing Rabi oscillations 
with some damping [13,14] [Fig. 2(c)]. The red line shown serves only as a guide to the eye. Power dependent damping of Rabi oscillations has been observed previously in quantum dots and possible mechanisms are the subject of theoretical investigations $[15,16]$. However, as we are primarily concerned with $\frac{\pi}{2}$ pulses, damping at higher powers is of little relevance to this work.

\section{A. Two-level system}

We use the setup illustrated in Fig. 2(d) to apply the Ramsey pulse sequence and record the emitted light intensity as a function of pulse separation. Scanning the position of the movable mirror of the Michelson interferometer allows us to control the pulse separation and to record the emitted light intensity after polarization filtering. Measurements are recorded using a single mode fiber coupled silicon avalanche photodiode. We observe a sinusoidal variation in the emission intensity [Figs. 2(f) and 2(g)] from which we extract the interference visibility at each pulse separation time [Fig. 2(e) in black].

The visibility decays exponentially with a decay time of $510 \pm 10$ ps. The decay time is close to the coherence time inferred from a linewidth measurement taken using a FabryPerot Etalon [605 $\pm(24 / 21)]$ ps, as expected from the WienerKhinchin theorem [17]. Figure 2(e) also shows the interference visibility between the laser excitation pulses (red). Due to this effect, oscillations shown in the gray region cannot be ascribed to RI. Outside of this region the low noise floor indicates that the oscillations are a result of RI.

\section{B. Four-level system}

Applying a magnetic field in the Faraday geometry removes the degeneracy between the spin-up and spin-down states resulting in four levels with distinct energies [Fig. 3(a)]. There are now two transitions allowed by the selection rules with an energy difference consistent with a combined electron and hole $g$ factor of $\left|g_{e}+g_{h}\right|=2.88 \pm 0.03$. Therefore, the diagonal transitions are suppressed relative to the vertical transitions and provide no noticeable contribution to the results at this laser energy. We set the laser energy between the two transition energies in order to excite both transitions equally [Fig. 3(c)].

Here we observe RI with the same decay time as at zero field [illustrated as a gray line in Fig. 3(b)], but with a beating pattern in the interference fringes [Fig. 3(b)]. The beat frequency corresponds to the energy difference between the allowed transitions. These results are explained by considering two independent two-level systems and can be represented as a Bloch sphere with two Bloch vectors. The two vectors precess about the $z$ axis at different rates and move in and out of phase with each other. When they are in phase we see a peak in visibility and when they are out of phase we see a minimum.

\section{Three-level system}

Setting the laser wavelength degenerate with one of the observed transitions in Fig. 3(c), we repeat the RI measurement. We again observe a slow beating pattern with high visibility, visible up until around 500 ps. However, this time we also observe a slow oscillation in the average intensity

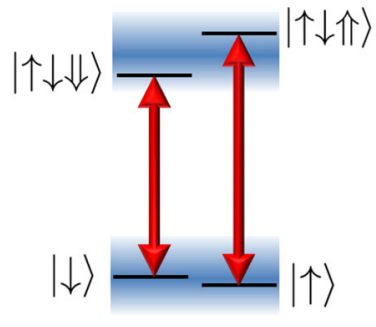

(a)

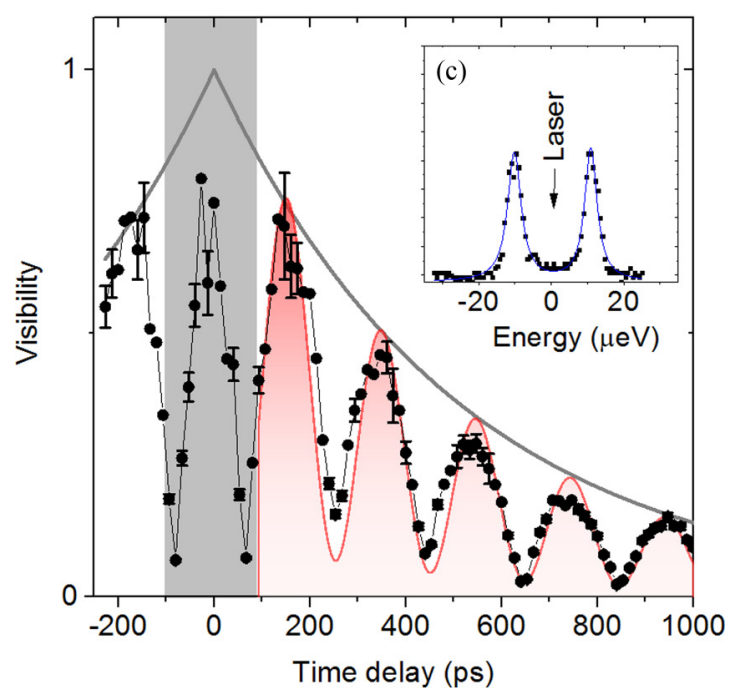

(b)

FIG. 3. (a) An illustration of the allowed transitions and energy levels in a Faraday geometry magnetic field. (b) A beating in the Ramsey fringe visibility at $125 \mathrm{mT}$ magnetic field. The gray exponential decay is a fit to the decay envelope of the two-level system as in Fig. 2(e). (c) Measured intensity and a function of laser detuning.

of the emitted light, which is most visible after 500 ps. Both the beating pattern and the slow oscillation have a frequency determined by the ground-state electron splitting. Here we discuss the mechanisms for both features.

The beat frequency corresponds to the energy of the electron Zeeman splitting. A field-dependent measurement allows us to determine the electron $g$ factor $\left(\left|g_{e}\right|=0.58 \pm 0.08\right)$ —in good agreement with $\left|g_{e}\right|=0.60 \pm 0.05$ made using the standard spectral method [18].

It is surprising that the ground state splitting can cause high visibility beats in the interferogram [Fig. 4(b)]. The graph appears to be a solid block of color due to the fast oscillations in $\mathrm{X}^{-}$intensity with pulse separation. The same is true of the simulated graphs in Figs. 4(c)-4(f). Although in an ideal dot the diagonal transition in Fig. 4(a) is forbidden, heavy-light hole mixing results in a weakly allowed diagonal transition [19-21]. The ground state splitting is rather small so the laser spectrum overlaps with both transitions, as illustrated in Fig. 4(a).

When considering a system where there is one vertical transition and one weakly allowed diagonal transition, we need to take the expected electron spin state into account. 


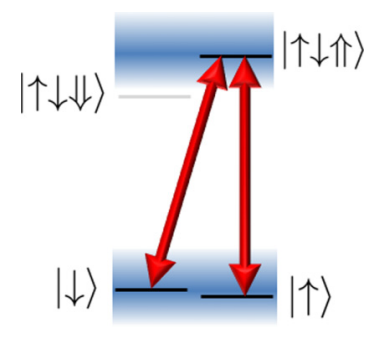

(a)

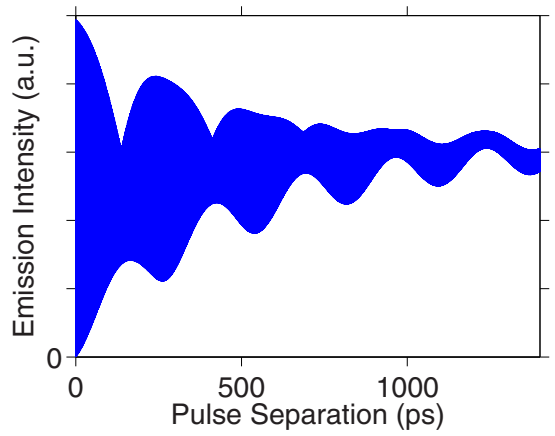

(d)

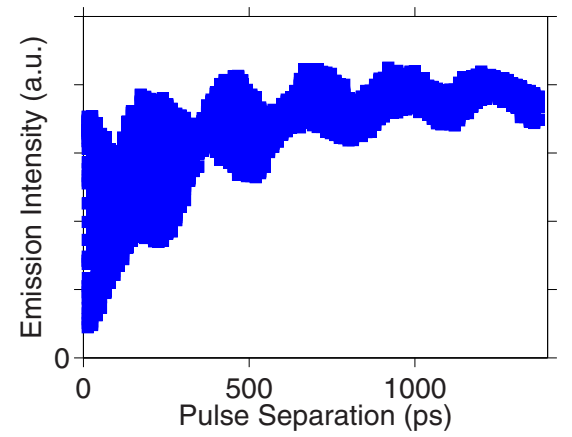

(b)

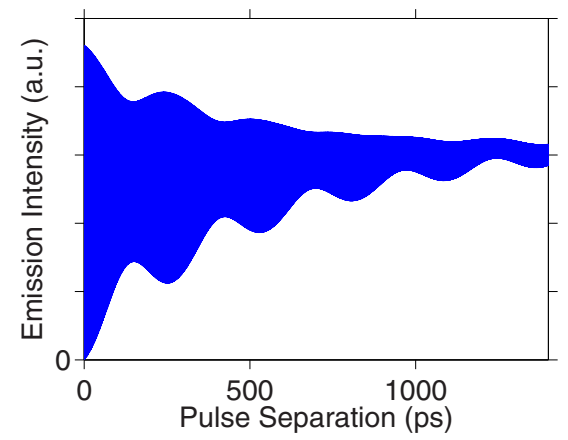

(e)

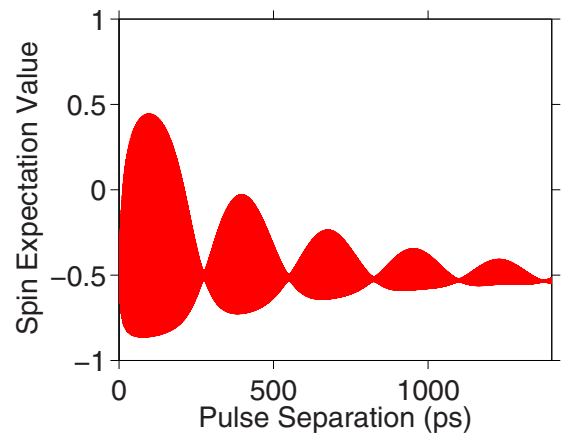

(c)

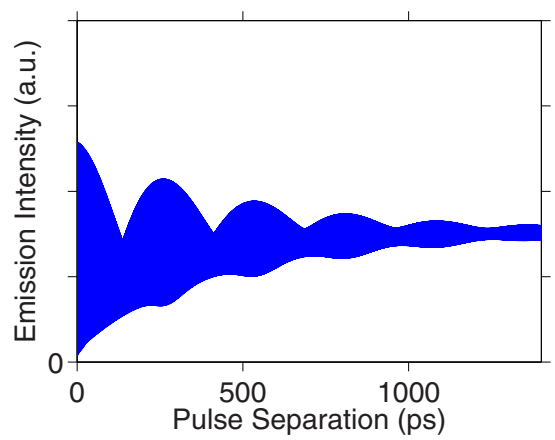

(f)

FIG. 4. (a) A sketch of the three-level system considered in the model. (b) The measured emission intensity of the three-level system as a function of pulse separation at $500 \mathrm{mT}$. (c) The expectation value of the ground state electron spin in the long time limit as a function of pulse separation. (d) A full simulation of the expected emission intensity as a function of pulse separation. (e) The same simulation as (d), but here the effect of the pulse separation-dependent spin preparation is neglected. (f) The same simulation as (d) but here the coherence terms between the two electron ground states are rapidly destroyed.

For example, the system is more likely to be excited when the electron is in the $|\uparrow\rangle$ state due to the stronger coupling of the vertical transition to laser field. Therefore, in order to model the three-level system we must calculate the expected initial state of the electron (see Sec. IV).

We find that the expected initial state of the electron is dependent on the pulse separation [Fig. 4(c)]. This dependence can be intuitively understood by considering weak pulses using a model with two Bloch vectors (note that this explanation neglects any coupling between the two low energy states via the excited state). After the first pulse, the Bloch vectors for the two transitions precess about the $z$ axis at different rates. When they are completely out of phase the second pulse can then destructively interfere with one transition and constructively interfere with the other. The result is as if we were preferentially driving the constructively interfering transition and only weakly driving the destructively interfering one. After many two-pulse repetitions, we see spin state preparation as one spin state is being preferentially driven into the excited state. This explains why the nodes in fringe visibility [Fig. 4(d)] correspond to antinodes in the spin-expectation value graph [Fig. 4(c)]. Including the spin preparation effect in the simulations allows us to recover a beat visibility that appears similar to the measured result [Fig. 4(d)]. In contrast, Fig. 4(e) is the result of ignoring the spin preparation-note the considerably lower beat visibility. We conclude that this pulse separation-dependent spin preparation is an important effect in this three-level system and may be of use in other lambda systems.

Next we turn our attention to the slow oscillation in the average emission rate visible in the interferogram, a feature which is particularly noticeable at 600-1400 ps [Fig. 4(b)]. This behavior is not seen in the two- or four-level systems. The oscillation frequency corresponds to the Larmor frequency of the electron. We determine that not only is the expected population of the electron spin state important for determining the emission intensity, the coherence matters too. The first pulse creates an impure coherent superposition of the electron spin state by coupling the two spin states via the excited state. The mechanism by which this superposition is generated (given in Sec. IV) is similar to that presented in [22], but here we use a Faraday geometry magnetic field rather than a Voigt geometry field. Our simulations predict the observed slow oscillation and show that the phase of the electron spin before the second pulse affects the emission intensity. To emphasize this point we destroy the electron coherence terms in the model and see that this removes the slow oscillatory behavior [Fig. 4(f)]. The model predicts that the visibility of these oscillations will be higher at higher pulse powers and this is observed in our measurements. To the best of our knowledge, this is the first demonstration of an optically induced coherent rotation of an electron spin state in a quantum dot in a Faraday geometry magnetic field. The coherent control of a trapped charge in a QD with Faraday geometry selection rules is a 
key capability for one of the proposed methods for generating photonic cluster states [23].

The simulations also indicate that the decay of these oscillations at long times is only dependent on the decay and dephasing times of the electron spin.

\section{THE MODEL}

The most interesting behavior observed in our work was in the "three-level" regime. In order to better understand our results we constructed a simulation in MATLAB.

We use the Lindblad master equation equation to model the evolution of the system:

$$
\dot{\rho}(t)=-\frac{i}{\hbar}[\mathcal{H}, \rho(t)]+\sum_{n} \gamma_{n} \mathcal{L}_{n}(\rho),
$$

where the $-\frac{i}{\hbar}[\mathcal{H}, \rho(t)]$ term describes the unitary evolution of density matrix $\rho$ under the Hamiltonian $\mathcal{H}$ according to the Liouville-Von Neumann equation and the $\sum_{n} \gamma_{n} \mathcal{L}_{n}(\rho)$ represents the nonunitary terms due to decays and environmental interactions. The $\mathcal{L}_{n}(\rho)$ are the decay and dephasing superoperators and the $\gamma_{n}$ give the decay and dephasing rates.

\section{A. Operator definitions}

\section{Free evolution}

A pure state of the three-level system is defined as

$$
|\psi\rangle=\alpha|\uparrow\rangle+\beta|\downarrow\rangle+\delta|\uparrow \downarrow \uparrow\rangle,
$$

and we define the density operator so that

$$
\rho_{11}=|\alpha|^{2}, \quad \rho_{22}=|\beta|^{2}, \quad \rho_{33}=|\delta|^{2} .
$$

The free evolution of the system is governed by the Hamiltonian

$$
\mathcal{H}_{\text {Evolution }}=\left(\begin{array}{ccc}
E_{\uparrow} & 0 & 0 \\
0 & E_{\downarrow} & 0 \\
0 & 0 & E_{\uparrow \downarrow \uparrow}
\end{array}\right),
$$

where $E_{\uparrow}$ is the energy of the electron spin-up state, $E_{\downarrow}$ is the energy of the electron spin-down state, and $E_{\uparrow \downarrow \uparrow}$ is the energy of the excited state with a hole spin of $+\frac{3}{2}$. The $|\uparrow \downarrow \Downarrow\rangle$ state is not considered-when using the laser detuning used in the three-level case we assume the system is not driven into this state due to the minimal overlap between the transitions to this state and the laser energy.

\section{Excitation}

The excitation and deexcitation operators between the two transitions are defined as

$$
\begin{aligned}
\sigma_{\uparrow, \downarrow}^{+} & =\left(\begin{array}{lll}
0 & 0 & 0 \\
1 & 0 & 0 \\
0 & 0 & 0
\end{array}\right), \quad \sigma_{\uparrow, \uparrow \downarrow \uparrow}^{+}=\left(\begin{array}{lll}
0 & 0 & 0 \\
0 & 0 & 0 \\
1 & 0 & 0
\end{array}\right), \\
\sigma_{\downarrow, \uparrow \downarrow \uparrow}^{+} & =\left(\begin{array}{lll}
0 & 0 & 0 \\
0 & 0 & 0 \\
0 & 1 & 0
\end{array}\right), \quad \sigma^{-}=\sigma^{+\dagger} .
\end{aligned}
$$

Making use of these operators to describe the effect of the laser pulse on the system we define the Hamiltonian:

$\mathcal{H}_{\text {Pulse }}=F_{\uparrow, \uparrow \downarrow \uparrow}\left(\sigma_{\uparrow, \uparrow \downarrow \uparrow}^{+}+\sigma_{\uparrow, \uparrow \downarrow \uparrow}^{-}\right)+F_{\downarrow, \uparrow \downarrow \uparrow}\left(\sigma_{\downarrow, \uparrow \downarrow \uparrow}^{+}+\sigma_{\downarrow, \uparrow \downarrow \uparrow}^{-}\right)$,

where $F_{\uparrow, \uparrow \downarrow \uparrow}$ and $F_{\downarrow, \uparrow \downarrow \uparrow}$ determine the strength of the interaction between the laser field and the transition (i.e., they account for both the laser power and the coupling strength between the transitions and the laser field).

\section{Decay and dephasing}

In order to account for radiative decay, the decay superoperators between states are defined as

$\mathcal{L}_{\text {decay }}(\rho(t))=\frac{1}{2}\left[2 \sigma^{-} \rho(t) \sigma^{+}-\rho(t) \sigma^{+} \sigma^{-}-\sigma^{+} \sigma^{-} \rho(t)\right]$.

Note that it is assumed that there is no decay between the electron spin states over the time scales investigated. (The device is held at a bias in the center of the charge plateau to maximize the electron spin lifetime.)

Next, in order to account for the dephasing due to environmental interactions, we define the pure dephasing operators as

$$
\mathcal{L}_{\text {deph }}(\rho(t))=-\left[\mathcal{I}_{a} \rho(t) \mathcal{I}_{b}+\mathcal{I}_{b} \rho(t) \mathcal{I}_{a}\right],
$$

where the elements of the matrix $\mathcal{I}_{a}$ are $\mathcal{I}_{a, n m}=\delta_{n, m} \delta_{n, a}$. $a$ and $b$ indicate the states between which the coherences are destroyed. For example, for $a=1, b=3$, the superoperator destroys the coherence terms between the $|\uparrow\rangle$ and $|\uparrow \downarrow \uparrow\rangle$ states. Removing the effect of the electron spin coherence on the result [as in Fig. 4(f)] is done by setting the electron dephasing $\gamma$ to a high value so that the coherences are destroyed almost immediately.

\section{B. Simulating free evolution and the Ramsey pulses}

\section{Free evolution}

For time-independent Liouvillians, Eq. (1) can be integrated to get

$$
\rho(t)=e^{\mathcal{L} t} \rho(0),
$$

where the Hamiltonian and all the relevant superoperators have been absorbed by $\mathcal{L}$.

A matrix representation of $\mathcal{L}$ is found by combining superoperators which perform left or right multiplication. The left and right superoperators are defined as

$$
\begin{aligned}
& L(M) \rho=M \rho \longrightarrow(M \otimes \mathbb{1}) \tilde{\rho}, \\
& R(M) \rho=\rho M \longrightarrow\left(\mathbb{1} \otimes M^{T}\right) \tilde{\rho},
\end{aligned}
$$

where $\tilde{\rho}$ is the flattened density matrix defined by the mapping

$$
\rho=\sum_{i} p_{i}\left|\psi_{i}\right\rangle\left\langle\psi_{i}\left|\longrightarrow \sum_{i} p_{i}\right| \psi_{i}\right\rangle \otimes\left|\psi_{i}\right\rangle^{*}=\tilde{\rho}
$$

\section{The pulse operator}

To simulate the effect of a resonant laser pulse, the pulse operator is defined using the pulse Hamiltonian as

$$
P=\exp \left[-\frac{i \pi}{2}\left(\mathcal{H}_{\text {Pulse }} \otimes \mathbb{1}-\mathbb{1} \otimes \mathcal{H}_{\text {Pulse }}^{T}\right)\right] \text {. }
$$


Note that $P$ is dependent on the field-oscillator couplings $F_{\uparrow, \uparrow \downarrow \uparrow}$ and $F_{\downarrow, \uparrow \downarrow \uparrow}$. It is assumed that the length of the pulse is short in relation to all the relevant dephasing and decay times, so no decay or dephasing terms are included in the pulse operator.

\section{The Ramsey pulse sequence}

To simulate the effect the Ramsey pulse sequence with pulses separated by time $t$ on the system, the operator $P e^{t \sum_{i} \mathcal{L}_{i}} P$ is applied. This operator represents a pulse followed by free evolution for a time $t$ followed by a second pulse. The flattened density operator of the system after the Ramsey pulse sequence for an initial flattened density matrix of $\tilde{\rho}(0)$ is given by

$$
\tilde{\rho}(t)=P e^{t \sum_{i} \mathcal{L}_{i}} P \tilde{\rho}(0),
$$

where $\sum_{i} \mathcal{L}_{i}$ represents a sum over all of the dephasing and decay operators as well as the superoperator that governs the free evolution under the system Hamiltonian.

\section{Determining the expected initial state $\tilde{\rho}(0)$}

It might seem that this is sufficient to simulate the experiment, however Eq. (13) is dependent on the state of the system before the pulse sequence $\tilde{\rho}(0)$, which has not yet been determined. In order to simulate the results of the experiment, $\tilde{\rho}(0)$ should be the expected state of the system before the Ramsey pulse sequence is applied. As the measured result used many millions of Ramsey pulse sequences per data point, it is reasonable to assume that the majority of the measurement takes place after the system has already been hit by many pulses. Given this, this subsection will focus on how the value of $\tilde{\rho}(0)$ is determined.

For a given pulse separation, $\tilde{\rho}(0)$ is defined as

$$
\tilde{\rho}(0)=\lim _{n \rightarrow \infty}\left[e^{T \sum_{i} \mathcal{L}_{i}} P e^{t \sum_{i} \mathcal{L}_{i}} P\right]^{n} \tilde{\rho}_{s} \equiv \lim _{n \rightarrow \infty} S^{n} \tilde{\rho}_{s},
$$

where $\tilde{\rho}_{s}$ is any state and $T$ is the remaining time between the two-pulse sequences (in our case $12.5 \mathrm{~ns}-t$ ). In other words, $\tilde{\rho}(0)$ is the expected state that the system tends to as it is hit by many Ramsey pulse sequences. Note that the electron spin is only allowed to dephase and not to decay. For the parameters used, there no electron spin coherences for the expected initial state $\tilde{\rho}(0)$ (the relevant elements of the density matrix have a magnitude of the order of $\left.10^{-11}\right)$.

In the simulations performed for this work an unexcited state with the electron in a maximally mixed state was used for $\tilde{\rho}_{s}$, but as would be expected intuitively, $\tilde{\rho}(0)$ is found to be independent of the choice of $\tilde{\rho}_{s}$ whenever both $F_{\uparrow, \uparrow \downarrow \uparrow}$ and $F_{\downarrow, \uparrow \downarrow \uparrow}$ are nonzero and the $\gamma$ values are set appropriately.

In order to determine the existence and the value of $\tilde{\rho}(0)$, it is necessary to find $\lim _{n \rightarrow \infty} S^{n}$. This is done by diagonalizing $S$ to get

$$
S=R D R^{-1},
$$

where $D$ is a diagonal matrix of eigenvalues $\lambda_{l}, R$ is a matrix of eigenvectors of $S$, and $R^{-1}$ is the inverse of $R$. This means that $S^{n}$ can be written as

$$
S^{n}=\left(R D R^{-1}\right)^{n}=R D^{n} R^{-1} .
$$

To make physical sense, the elements of $D^{n}$ should not diverge as $n \rightarrow \infty$, therefore it is expected that $\forall l:\left|\lambda_{l}\right| \leqslant 1$. Similar physical arguments also suggest that $D^{n}$ should not converge as $n \rightarrow \infty$, so it is also expected that $\exists l:\left|\lambda_{l}\right|=1$.

As $\forall\left|\lambda_{l}\right|<1: \lim _{n \rightarrow \infty} \lambda_{l}^{n}=0$, all the elements for which $\left|D_{l m}\right|<1$ are set to 0 , leaving a diagonal matrix $D^{\prime}$ with elements that have absolute values of 0 or 1 . Therefore, all of the remaining diagonal elements are either $D_{l l}^{\prime}=0$ or $D_{l l}^{\prime}=e^{i \phi}$. If any nonzero $D_{l l}^{\prime} \neq 1$ then $\lim _{n \rightarrow \infty} D^{\prime n}$ cannot be determined as the element will simply precess about the Argand plane as $n$ increases and so there may not be a $\rho_{s}$ independent $\rho(0)$. This is found to be the case when there are no decay or dephasing terms included in the simulation. For all of the matrices considered in this work, all nonzero values of $D_{l l}^{\prime}$ were 1 , meaning that $D$ and $S$ are semiconvergent matrices. Therefore,

$$
\lim _{n \rightarrow \infty} D^{n}=\lim _{n \rightarrow \infty} D^{\prime n}=D^{\prime}
$$

So

$$
\lim _{n \rightarrow \infty} S^{n}=R D^{\prime} R^{-1}
$$

This means that the operator $R D^{\prime} R^{-1}$ acting on any state gives the expected state of the system after many Ramsey pulse sequences and so the expected initial state is given by

$$
\tilde{\rho}(0)=R D^{\prime} R^{-1} \tilde{\rho} .
$$

Once $\tilde{\rho}(0)$ has been found for a given pulse separation, the Ramsey pulse sequence operator can be applied to determine the effect of the Ramsey pulse sequence and to extract the probability of the system being in an excited state [with the mapping used here this is given by the term $\left.\tilde{\rho}(t)_{9}\right]$, which is proportional to the expected intensity of the emitted light.

When we neglect this spin pulse separation-dependent effect we perform the same process but only include one laser pulse per cycle rather than the two Ramsey pulses. This removes the pulse separation dependence of the expected initial state.

\section{The generation of a superposition of electron spin states}

We claim that the coherent superposition of the electron spin states is a result of the coupling of the two low energy electron states via the excited state. We also claim that this effect is more visible at higher powers. Here we briefly explain the mechanism.

Unitary evolution under the pulse operator can be described by the operator

$$
e^{-\frac{i \mathcal{H}_{\text {Pulse }} t}{\hbar}}=\sum_{k=0}^{\infty} \frac{1}{k !}\left(-i \frac{\mathcal{H}_{\text {Pulse }} t}{\hbar}\right)^{k},
$$

where $t$ is the length of the pulse. To second order this is

$$
e^{-\frac{i \mathcal{H}_{\text {Pulset }}}{\hbar}} \approx-i \frac{\mathcal{H}_{\text {Pulse }} t}{\hbar}-\frac{1}{2} \frac{\mathcal{H}_{\text {Pulse }}^{2} t^{2}}{\hbar^{2}} .
$$

Therefore, for short times or for low pulse powers, the first order term will have the biggest effect and the second order 


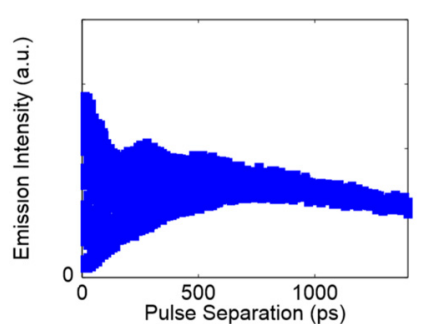

(a)

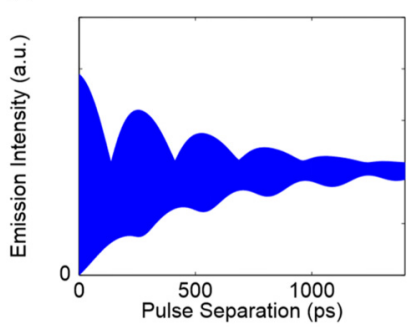

(c)

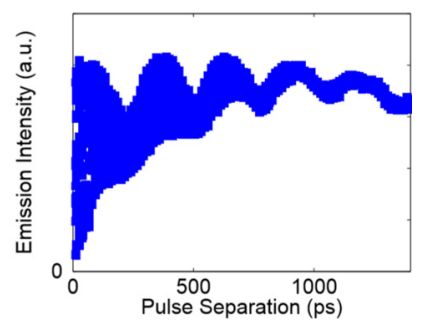

(b)

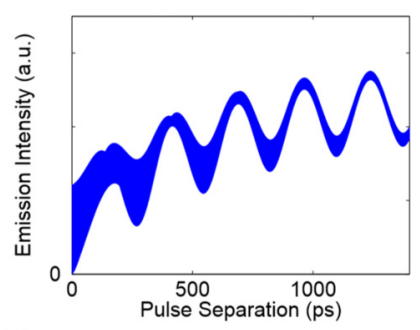

(d)

FIG. 5. (a) Experimental measurement of Ramsey fringes at half the laser field amplitude used in Fig. 4. (b) Experimental measurement of Ramsey fringes at twice the laser field amplitude used in Fig. 4. (c) A simulation of (a). (d) A simulation of (b).

term will be small. $\mathcal{H}_{\text {Pulse }}$ is given by

$$
\mathcal{H}_{\text {Pulse }}=\left(\begin{array}{ccc}
0 & 0 & F_{\uparrow, \uparrow \downarrow \uparrow} \\
0 & 0 & F_{\downarrow, \uparrow \downarrow \uparrow} \\
F_{\uparrow, \uparrow \downarrow \uparrow} & F_{\downarrow, \uparrow \downarrow \uparrow} & 0
\end{array}\right),
$$

so for low pulse powers there should be very little coupling between the $|\uparrow\rangle$ and $|\downarrow\rangle$ states. In other words, low pulse powers should not prepare a coherent superposition of the trapped electron spin state. (The elements $\mathcal{H}_{\text {Pulse,12 }}$ and $\mathcal{H}_{\text {Pulse,21 are } 0 .)}$

The second order term is proportion proportional to

$$
\mathcal{H}_{\text {Pulse }}^{2}=\left(\begin{array}{ccc}
F_{\uparrow, \uparrow \downarrow \uparrow}^{2} & F_{\uparrow, \uparrow \downarrow \uparrow} F_{\downarrow, \uparrow \downarrow \uparrow} & 0 \\
F_{\uparrow, \uparrow \downarrow \uparrow} F_{\downarrow, \uparrow \downarrow \uparrow} & F_{\downarrow, \uparrow \downarrow \uparrow}^{2} & 0 \\
0 & 0 & F_{\uparrow, \uparrow \downarrow \uparrow}^{2}+F_{\downarrow, \uparrow \downarrow \uparrow}^{2}
\end{array}\right),
$$

which does couple between the $|\uparrow\rangle$ and $|\downarrow\rangle$ states (elements $\mathcal{H}_{\text {Pulse,12 }}$ and $\mathcal{H}_{\text {Pulse,21 }}$ are nonzero) and can prepare a coherent superposition. It is therefore expected that the slow oscillation should be more visible with higher pulse powers when the second order term has a greater effect.

Figure 5 shows the interference fringes recorded at half the laser field amplitude and twice the laser field amplitude used in Sec. III C. Simulations of these measurements are also presented.

Although these simulations reproduce many of the features seen in the power dependent measurements, they do not resemble the measured data as closely as the simulations shown earlier. The beating pattern is visible for both high and low powers in the measured and simulated results, whereas the slow oscillation dominates at higher powers. This is in agreement with what is expected from considering the first and second order terms of the pulse operator.

\section{E. Model parameters}

The purpose of the model is to reproduce the rich variety of beats and oscillations observed in the measured data for realistic input parameters.

Some of the parameters of the model were set by an external factor (such as the magnetic field) and some could be easily measured (such as the transition energy). For the remaining parameters we used realistic estimates based on prior work.

\section{Parameters with values extracted from measurements and external factors}

The energy separation of the two electron spin states was calculated using the applied magnetic field $(0.5 \mathrm{~T})$, the measured electron $g$ factor (0.58), and the Bohr magneton $\left(E=B g \mu_{B}\right)$.

The energy of the excited state was found using a spectrometer to record the wavelength of the emitted photon allowing us to calculate the transition energy.

The $\gamma$ decay values are extracted from exciton lifetime measurement (959 ps). However, as there are two possible decay routes, the $\gamma$ values are set such that the difference in decay rates is equal to the difference in transition strengths (the difference in transition strengths is estimated based on prior research).

The $\gamma$ dephasing values for the exciton are set so that coherence time of the exciton matches the coherence time inferred from the linewidth measurement (600 ps).

\section{Parameters with estimated values based on prior work}

We do not have a measurement for the coherence time of the electron spin, but prior work indicates that the coherence time of the electron spin is considerably longer than the coherence time of the exciton, so we set the electron spin coherence time to be 5000 ps, roughly 10 times the coherence time of the exciton.

The forbidden transitions are allowed by heavy-light hole mixing, so we set the transition strength ratio between the allowed and forbidden transitions to be 5:1, in accordance with prior work [24]. However, this does not determine how strongly we are driving each of the transitions. Sweeping the laser energy across the transitions, we see two peaks in emitted intensity that each correspond to a pair of transitions (one allowed and one forbidden). For the three-level system experiments, the laser energy was set to one of the peaks. Clearly there is some uncertainty about the correct values of $F_{\uparrow, \uparrow \downarrow \uparrow}$ and $F_{\downarrow, \uparrow \downarrow \uparrow}$ for our system. In the simulations shown in Sec. III C we used $F_{\uparrow, \uparrow \downarrow \Uparrow}=0.355, F_{\downarrow, \uparrow \downarrow \uparrow}=0.085$ based on the results of brightness as a function of laser power and detuning simulation and on the similarity between the Ramsey interference simulation and the measured results for these parameters.

\section{CONCLUSION}

Our study of RI in the three-level system demonstrates that we can prepare a spin by controlling the time delay between successive pulses. This shows that it is possible to use a single pulsed laser for both spin state preparation and exciton creation, in contrast to work such as [25] which makes use 
of multiple lasers to address the spin and exciton populations separately. Measurements of the decay of the slow oscillation visible in Fig. 4(b) at longer delay times than are possible with our setup should allow direct measurement of the electron spin coherence time. The results also show that it is possible to manipulate the state of the system by coherently driving the forbidden diagonal transitions of a QD in the Faraday geometry with a resonant laser pulse. Finally, we note that the ability to prepare and coherently manipulate an electron spin state, along with improvements in collection efficiency (e.g. [26]) are key capabilities required to generate electron spin-photon frequency entanglement [27].

\section{DATA ACCESS}

All experimental data used in this paper is publicly available and can be found at https://www.repository. cam.ac.uk/handle/1810/253343

\section{ACKNOWLEDGMENTS}

The authors acknowledge funding from the EPSRC for the MBE system used in the production of the QD-LED. J.L. gratefully acknowledges financial support from the EPSRC CDT in Photonic Systems Development and Toshiba Research Europe Ltd.
[1] L. Essen and J. Parry, Nature (London) 176, 280 (1955).

[2] S. Haroche and J. M. Raimond, Exploring the Quantum (Oxford University Press, Oxford, 2006).

[3] A. Ramsay, Semicond. Sci. Technol. 25, 103001 (2010).

[4] H. Jayakumar, A. Predojević, T. Huber, T. Kauten, G. S. Solomon, and G. Weihs, Phys. Rev. Lett. 110, 135505 (2013).

[5] G. De Lange, Z. Wang, D. Riste, V. Dobrovitski, and R. Hanson, Science 330, 60 (2010).

[6] K. Litvinenko, E. Bowyer, P. Greenland, N. Stavrias, J. Li, R. Gwilliam, B. Villis, G. Matmon, M. Pang, B. Redlich et al., Nat. Commun. 6, 6549 (2015).

[7] P. Bianucci, A. Muller, C. K. Shih, Q. Q. Wang, Q. K. Xue, and C. Piermarocchi, Phys. Rev. B 69, 161303 (2004).

[8] J. Taylor, P. Cappellaro, L. Childress, L. Jiang, D. Budker, P. Hemmer, A. Yacoby, R. Walsworth, and M. Lukin, Nat. Phys. 4, 810 (2008).

[9] N. H. Bonadeo, J. Erland, D. Gammon, D. Park, D. Katzer, and D. Steel, Science 282, 1473 (1998).

[10] S. Stufler, P. Ester, A. Zrenner, and M. Bichler, Phys. Rev. Lett. 96, 037402 (2006).

[11] C. Bonato, M. S. Blok, H. T. Dinani, D. W. Berry, M. L. Markham, D. J. Twitchen, and R. Hanson, Nature Nanotech., doi: 10.1038/nnano.2015.261.

[12] J. Johansson, P. Nation, and F. Nori, Comput. Phys. Commun. 184, 1234 (2013).

[13] R. Melet, V. Voliotis, A. Enderlin, D. Roditchev, X. L. Wang, T. Guillet, and R. Grousson, Phys. Rev. B 78, 073301 (2008).

[14] E. Flagg, A. Muller, J. Robertson, S. Founta, D. Deppe, M. Xiao, W. Ma, G. Salamo, and C.-K. Shih, Nat. Phys. 5, 203 (2009).
[15] A. J. Ramsay, A. V. Gopal, E. M. Gauger, A. Nazir, B. W. Lovett, A. M. Fox, and M. S. Skolnick, Phys. Rev. Lett. 104, 017402 (2010).

[16] D. Mogilevtsev, A. P. Nisovtsev, S. Kilin, S. B. Cavalcanti, H. S Brandi, and L. E. Oliveira, Phys. Rev. Lett. 100, 017401 (2008).

[17] E. Skantzakis, P. Tzallas, J. E. Kruse, C. Kalpouzos, O. Faucher, G. Tsakiris, and D. Charalambidis, Phys. Rev. Lett. 105, 043902 (2010).

[18] A. Tartakovskii, T. Wright, A. Russell, V. Fal'Ko, A. Van'kov, J. Skiba-Szymanska, I. Drouzas, R. Kolodka, M. Skolnick, P. Fry et al., Phys. Rev. Lett. 98, 026806 (2007).

[19] A. Delteil, W. B. Gao, P. Fallahi, J. Miguel-Sanchez, and A. Imamoğlu, Phys. Rev. Lett. 112, 116802 (2014).

[20] A. V. Koudinov, I. A. Akimov, Y. G. Kusrayev, and F. Henneberger, Phys. Rev. B 70, 241305 (2004).

[21] T. Belhadj, T. Amand, A. Kunold, C.-M. Simon, T. Kuroda, M. Abbarchi, T. Mano, K. Sakoda, S. Kunz, X. Marie, and B. Urbaszek, Appl. Phys. Lett. 97, 051111 (2010).

[22] S. E. Economou and T. L. Reinecke, Phys. Rev. Lett. 99, 217401 (2007).

[23] N. H. Lindner and T. Rudolph, Phys. Rev. Lett. 103, 113602 (2009).

[24] Y. Cao, A. J. Bennett, I. Farrer, D. A. Ritchie, and A. J. Shields, Phys. Rev. B 92, 081302 (2015).

[25] D. Press, T. D. Ladd, B. Zhang, and Y. Yamamoto, Nature (London) 456, 218 (2008).

[26] A. J. Bennett, J. P. Lee, D. J. P. Ellis, T. Meany, E. Murray, F. Floether, J. P. Griffths, I. Farrer, D. A. Ritchie, and A. J. Shields, arXiv:1508.01637.

[27] W. B. Gao, P. Fallahi, E. Togan, J. Miguel-Sanchez, and A. Imamoglu, Nature (London) 491, 426 (2012). 